\title{
Cerebrospinal fluid immunoglobulin quotients, kappa/lambda ratios, and viral antibody titres in neurological disease
}

\author{
P. J. ROBERTS-THOMSON ${ }^{1}$, M. M. ESIRI ${ }^{2}$, A. C. YOUNG ${ }^{3}$, AND $^{2}$ \\ I. C. M. MACLENNAN ${ }^{1}$
}

From the Nuffield Department of Clinical Medicine ${ }^{1}$, and Department of Neuropathology ${ }^{2}$, Radcliffe Infirmary, Oxford $O X 26 \mathrm{HE}$, and Department of Clinical Neurology, The Churchill Hospital, Headington, $\mathrm{Oxford}^{3}$

SYNOPSIS A description has been given of cerebrospinal fluid (CSF) immunoglobulins in 355 patients with demyelinating, infectious, neuropathic, and other neurological disorders. An increase in the CSF IgG/albumin quotient was observed in 19/36 (53\%) cases of definite multiple sclerosis (MS), in $13 / 47(28 \%)$ cases of probable or possible MS, in $6 / 9(67 \%)$ cases of proven herpes simplex viral encephalitis (HSVE), in $3 / 4(75 \%)$ cases of neurosyphilis, in $1 / 1$ case of subacute sclerosing panencephalitis (SSPE), in 2/9 (22\%) cases of other central nervous system infections, and in $2 / 12(17 \%)$ cases of polyneuritis when compared with a group of 236 patients having other neurological disorders. In contrast, a relative increase in the CSF IgA or IgM was seen only in some of the patients with central nervous system infections.

It was also found that the quotient CSF/serum IgG, expressed as a percentage of the CSF/serum albumin, was better in distinguishing patients with definite or suspected MS from those with other neurological disorders than the quotients IgG/albumin or IgG/total protein.

The CSF $\kappa / \lambda$ ratio and the CSF and serum complement-fixing antibody titre to measles and herpes simplex virus were measured in many of the patients. In general, abnormalities of these measurements were associated with raised IgG/albumin quotients. However, in eight patients with definite or suspected MS, a normal IgG/albumin quotient was found with abnormal CSF $\kappa / \lambda$ ratios ( 6 cases) or abnormal CSF titres of measles antibody ( 7 cases). In addition, two patients with HSVE had normal IgG/albumin ratios but detectable herpes antibody in the CSF.

These findings suggest that the measurement of the relative concentration of CSF immunoglobulin in combination with the $\kappa / \lambda$ ratio and antibody titre to various viruses may supplement each other in the endeavour to detect central nervous system immunoglobulin synthesis in neurological diseases.

In normal individuals, immunoglobulin in the CSF is derived from the plasma by diffusion across the blood/CSF barrier (see Schultze and Heremans (1966) Chapter 3 for review). Rosenthal and Soothill (1962) have shown that the quantity of this CSF immunoglobulin is dependent on the serum immunoglobulin concentration, the molecular size of the immunoglobulin, and the permeability of the blood/ CSF barrier. Thus for healthy individuals IgM is totally excluded from the CSF owing to its large size and low plasma concentration but may be present in conditions in which a gross defect in the
blood/CSF barrier has occurred or where a high serum level exists.

However, in the last two decades a considerable amount of clinical and experimental evidence has accumulated which strongly suggests that immunoglobulin may be additionally synthesized in the central nervous system (CNS) in certain demyelinating and infectious neurological diseases (reviewed by Tourtellotte (1970)).

The CNS-produced immunoglobulin may be indirectly assessed by measuring the CSF immunoglobulin. However, in doing this it is necessary to correct for the permeability of the blood/CSF barrier in order to account for the immunoglobulin derived 1105 
from the plasma. Tourtellotte (1970) and others have shown that the permeability of the blood/CSF barrier is accurately reflected by the CSF total protein or albumin level, and thus most investigators have related the absolute CSF immunoglobulin level to the CSF total protein or albumin level in order to derive a relative immunoglobulin concentration. If this relative concentration (or immunoglobulin quotient) is raised, this has been taken to indicate the local synthesis of immunoglobulin within the CNS.

The aim of the present study has been to measure the CSF immunoglobulin/albumin quotient in patients suffering from a variety of neurological disorders and to compare groups of patients with demyelinating, infectious, and polyneuritic diseases with the remaining patients. An endeavour has also been made to find which CSF quotient, out of several which have been described, is best at distinguishing the different groups. In addition, in certain patients the IgG/albumin quotient has been compared with the CSF kappa/lambda ratio and the CSF measles, mumps, and herpes simplex antibody titres to see the relationship between these measurements and to determine whether a further refinement in the separation between these diagnostic groups can be obtained.

\section{Patients}

The CSF was obtained from 355 patients suffering from a variety of neurological disorders. At the end of the period of study the clinical and laboratory details were reviewed from the case notes and a comparison was made with the CSF findings.

There were 236 patients who formed the neurological control group. They consisted of 92 females and 141 males ( 3 were unknown) and had an average age of 46.4 years. Thirty-eight of these patients had cerebrovascular disease, 14 degenerative disease, 17 epileptic disorders, 17 senile dementias, 41 cervical and lumbar osteopathies, $18 \mathrm{CNS}$ tumours, 26 neuroses or different forms of headache, 53 had other disorders, and 12 were undiagnosed.

There were 83 patients who had or were suspected of having MS. These patients were subdivided into two groups-the first consisting of patients with definite MS and the second of patients with probable or possible MS. This subdivision was, in general, based on the diagnostic criteria of McAlpine et al (1972). Thus patients with definite MS had evidence of multiple sites of CNS involvement associated with a history of a previous acute neurological episode followed by improvement and then one or more relapses. There were 23 females and 13 males in this subdivision; their mean age was 36.7 (range 17-60) years, the mean duration of the disease was 5.8 years (range 1 week-28 years), and the mean: number of neurological episodes was $3 \cdot 1$ (range 2-6) There were 25 females and 22 males with probables or possible MS. The mean age was $40 \cdot 7(12-64 \pm$ years, the mean duration 3.7 years (1 week-2 3 . years), and the mean number of attacks 1.8 (1-8) For both MS groups over $80 \%$ of the patients were studied during an active phase of the disease. The remaining patients were either in clinical remission or had the chronic progressive form of the disease. $\overrightarrow{\vec{\omega}}$

Twenty-four patients suffered from a CNS infeco tion. Patients were not included in this category un? less an infectious agent was positively identified of strongly implicated. Nine patients had HSVE, the diagnosis being proven by viral isolation from brain biopsy or CSF, positive immunofluorescence, or $\vec{a}$ rising titre to herpes simplex virus (HSV) in both ther serum and CSF. Five patients had bacterial menin? gitis, four patients had neurosyphilis, two patients had tuberculous meningitis, two patients had herpes: zoster meningoencephalitis, and one patient eacl\$ had SSPE and a cerebral abscess.

The remaining 12 patients had a polyneuropathy including four with characteristic features of the Guillain-Barré syndrome.

\section{Methods}

CEREBROSPINAL FLUID CSF was obtained by the lumbar route in all patients These specimens consisted of the majority received by the laboratory over a one-year period. However, seven specimens from patients with HSVE had been collected and stored at $-20^{\circ} \mathrm{C}$ over an eight-year period. Specimens of CSF were eliminated if heavily. bloodstained ( $>2000$ red blood cells $/ \mathrm{mm}^{3}$ ). The CSF cell count was routinely performed and thecells were separated by gentle centrifugation. The total protein was then measured and the specimens were stored at $-20^{\circ} \mathrm{C}$ for future protein estima tion. This was performed in large batches within sixg months of storage.

Serum was available for study from 62 of the patients. It was collected at the time of the lumbar puncture and studied simultaneously with its cor ${ }^{\circ}$ responding CSF.

CSF TOTAL PROTEIN

The CSF total protein was measured using a modifiso cation of the U-V spectrophotometric method ofD Waddell (1956). The CSF extinction at 215 and $225^{5}$ $\mathrm{m} \mu$ was measured and compared with a standard protein preparation and the mean of the two readings was calculated. 


\section{PROTEIN MEASUREMENTS}

CSF and serum albumin, IgG, IgA, IgM, and CSF $\kappa / \lambda$ ratio were measured using the single radial immunodiffusion method of Mancini et al (1965). Plates were prepared using Wellcome antihuman IgG, IgA, and IgM, Behringwerke antihuman albumin, and Dakapatts antihuman $\kappa$ and $\lambda$. The World Health Organisation reference serum $66 / 97$ was used as a standard for the immunoglobulin levels (and converted to $\mathrm{mg} \%$ using the formulae of Humphrey and Batty (1974) and for the $\kappa / \lambda$ ratio (where it was arbitrarily assigned a ratio of $1 \cdot 35$ ). Behringwerke standard serum no. 1730 was used as a standard for albumin.

The lowest limit at which immunoglobulin could be accurately measured using this method was 0.05 $\mathrm{iu} / \mathrm{ml}$ for IgG and IgA and $0 \cdot 1 \mathrm{iu} / \mathrm{ml}$ for IgM. The coefficient of variation for the measurement of albumin and $\mathrm{IgG}$ was less than $10 \%$. Each value for albumin and IgG was the mean of duplicate measurements while only single estimations were performed for the other proteins.

\section{VIRAL ANTIBODIES}

Complement fixing antibodies against measles, mumps, and HSV were measured in serum and CSF using the method of Smith et al (1967). If possible the $\mathrm{CSF} /$ serum antibody titre ratio was used in the assessment of antibody in the CSF. In CSF associated with an intact blood/CSF barrier no antibodies against these viruses are normally detected by this method.

\section{ANALYSIS OF RESULTS}

Protein concentrations in the healthy population approximate to a normal distribution when plotted on a logarithmic scale (Weeke and Krasilnikoff, 1970). In addition, a close approximation to a normal distribution was obtained for the IgG/albumin quotient and kappa/lambda ratio of the neurological control group when plotted on a log scale. Accordingly, all quotients have been plotted using this scale and the means and standard deviations calculated from the $\log$ of the quotients. Correlation coefficients between two variables have been calculated on the log measurements using the method of least squares.

\section{Results}

IgG/ALBUMIN QUOTIENT (see fig 1)

\section{Neurological control group}

The mean +2 standard deviations $(\mathrm{M}+2 \mathrm{SD})$ of the CSF IgG/albumin quotients obtained for 236 patients in this group was $13.9+14 \%$ (fig 1 ).
Six patients had a quotient greater than the $\mathbf{M}+$ 2SD. Two of these had long-standing dementia, and in one an elevated $\kappa / \lambda$ ratio (vide infra) and a mild CSF pleocytosis (6 lymphocytes/ $\mathrm{mm}^{3}$ ) were also found. A third patient had a low CSF albumin but a normal CSF IgG concentration.

Of the 10 patients with quotients less than the M - 2SD (6.9\%, fig 1), two were children with hydrocephalus. A third infant of 2 months of age was also noted with a low quotient and predictably had low levels of serum and CSF IgG. The remaining seven patients had a variety of unrelated diseases.

The $\mathrm{M}+2 \mathrm{SD}$ of the IgG/albumin quotients of a group of 36 patients suffering from myelographically defined cervical spondylosis with myelopathy or lumbar disc herniation was $14.5+10 \cdot 2 \%$. If $24 \cdot 7 \%$ is taken as an upper limit for this latter group, this is similar to the value $(27.9 \%)$ obtained for all the neurological control patients combined.

\section{Patients with multiple sclerosis}

The IgG/albumin quotient was elevated in 19/36 $(53 \%)$ of definite and $13 / 47(28 \%)$ of probable or possible cases of MS when compared with the $\mathbf{M}+$ 2SD of the neurological control group (fig 1).

The $M+$ SD for the mononuclear leucocyte cell counts in the CSF for patients with definite MS was 12.5 (range $0-81 / \mathrm{mm}^{3}$ ) and for patients with suspected MS, 4.6 $\left(0-21 / \mathrm{mm}^{3}\right)$. Twenty-one out of 34 $(62 \%)$ of the definite MS patients and 15/39 (39\%) of the suspected MS patients had CSF mononuclear leucocyte counts in excess of 5 cells $/ \mathrm{mm}^{3}$. A significant correlation was seen between this cell count and the IgG/albumin quotient when both MS groups were combined $(r=0.38, P=0.01)$ but not for the definite MS group taken alone. Significant correlations were also seen for both groups combined between the IgG/albumin quotient and the duration of the disease $(r=0.22, P=0.05)$, and the reciprocal of the albumin level $(r=0.24, P=0.04)$. These correlations were not significant, however, in the definite MS group alone. No correlation was observed with the age of the patients in either group or in both groups combined.

\section{Patients with CNS infection}

Several CSF specimens from patients comprising the CNS infection group had elevated IgG/albumin quotients (fig 1). Of interest was the finding that $6 / 9$ patients with proven HSVE had elevated quotients. Of the three CSF specimens with normal quotients, one was from a patient who had survived the encephalitic episode for over a year while another was from a patient whose encephalitis had been present for only two days. The clinical details of the third patient were unobtainable. The CSF of the remain- 


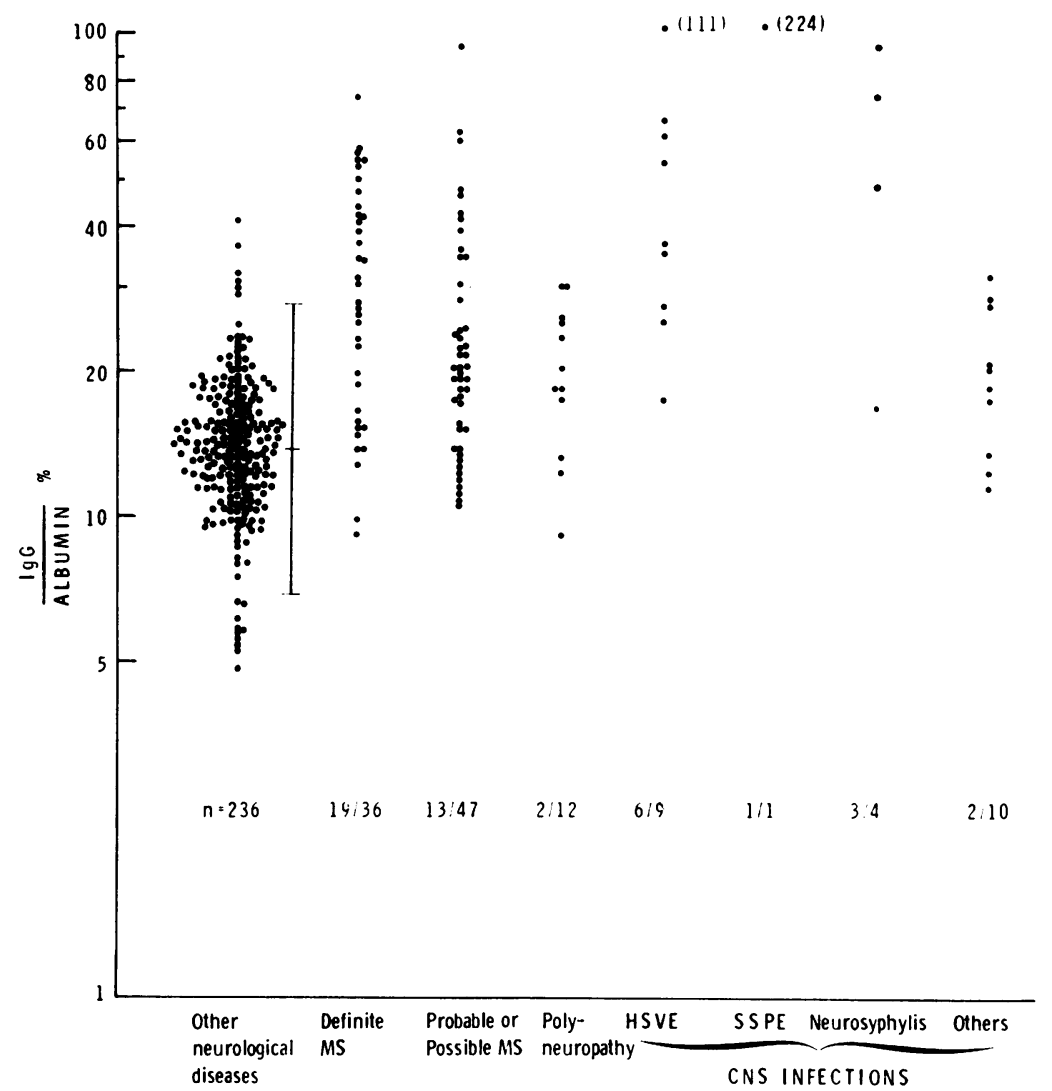

Fig 1 IgG/albumin quotient (expressed as a percentage) obtained for 355 patients with various neurological diseases. The vertical line represents the mean \pm 2 standard deviations $(M \pm 2 S D)$ of the quotient for the neurological control group. The $M+2 S D$ has been taken as the upper limit in the comparison of the various groups. The proportion of patients with elevated quotients has been indicated for each diagnostic group.

ing six patients were all obtained between the 12 th and the 200th day of the illness.

Three out of four patients with neurosyphilis had elevated quotients. The single patient with a normal quotient had been twice treated for tabes dorsalis over a 20-year period. Two other patients were untreated while the remaining patient had been diagnosed and treated seven years previously.

Three other patients, one with a cerebral abscess, one with SSPE, and one with bacterial meningitis, had elevated quotients.

\section{Patients with polyneuropathies}

Two patients out of 12 in this group showed an elevated IgG/albumin quotient (fig 1). In one of these a small monoclonal IgG band was noted in the corresponding serum.

QUOTIENT USED TO ADJUST FOR VARIATIONS IN BLOOD/CSF PERMEABILITY AND IN SERUM LEVELS OF IgG AND ALBUMIN (fig 2)

$A$ high CSF IgG/albumin ratio may merely reflect a high plasma level of IgG or, alternatively, a low plasma albumin level. To correct for variations in the 3 plasma levels of these patients a refinement in the CSF IgG/albumin quotient was used. This was the quotient CSF IgG/serum IgG (expressed as a percentage of the CSF/serum albumin ratio). The aim in using this quotient was to see if a better discrimination could be obtained between a neurological control group and a group of patients with MS. The relationship between this quotient and the quotients IgG/albumin and IgG/total protein is shown in fig 2 , together with the proportion of values in the MS? group which were above the $M+2 S D$ of the corresponding quotient in the other neurological disease $N$ group. All three groups have been subdivided, depending on whether the CSF total protein was above or below $75 \mathrm{mg} \%$. Thus it is seen in this figure $\omega$ that the upper limit for the neurological controlo group was higher if a breach in the blood/CSFo barrier had occurred. Comparison of these three quotients shows that the proportion of MS patients:with an elevated IgG/albumin or IgG/total protein ${ }_{-}^{-}$ quotient is less than that seen using the third $\frac{\mathrm{D}}{\mathrm{D}}$ quotient. 


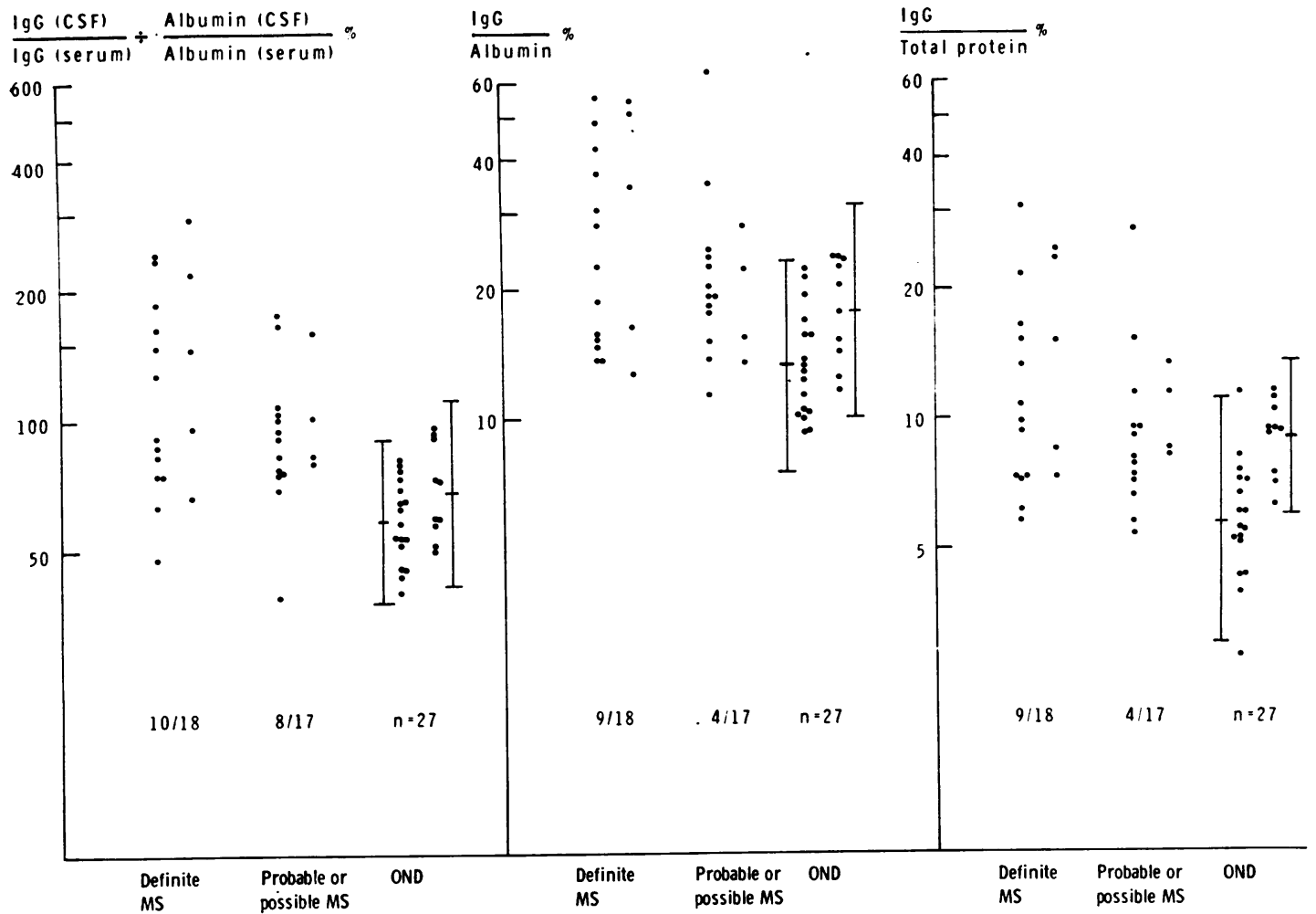

Fig 2 The comparison of three different quotients derived for the same group of 35 patients with multiple sclerosis (MS) when compared with 27 patients with other neurological diseases $(O N D)$. Each group has been subdivided into two, depending on whether the CSF total protein was normal (left bracket of each group) or greater than $75 \mathrm{mg} \%$ (right bracket of each group). In comparing the three different quotients, note the higher proportion of multiple sclerosis patients with an elevated CSF/serum IgG $\div$ CSF/serum albumin quotient. This suggests that this latter quotient is more discriminating in distinguishing patients with multiple sclerosis from those with other neurological disorders.

KAPPA/LAMBDA RATIO (fig 3)

Recent evidence has shown a preferential increase in the kappa light chain subclass of CSF IgG in some patients with MS (Zettervall and Link, 1970). Consequently the CSF kappa/lambda ratio was measured for many of the patients in the present study.

The one grossly elevated value in the neurological control group was from the patient with dementia, who also had an elevated IgG/albumin quotient (vide supra).

An elevated $\kappa / \lambda$ ratio was observed in $10 / 30$ (33\%) patients with definite MS and 10/38 (26\%) patients with possible or probable MS when compared with the neurological control group.

Elevated $\kappa / \lambda$ ratios were also seen in one patient with SSPE, two patients with HSVE, and one patient with tuberculous meningitis (fig 3).

With the exception of six MS patients, an elevated $\kappa / \lambda$ ratio was always associated with a raised $\mathrm{IgG} /$ albumin quotient (fig 4).

\section{IgA/ALBUMIN QUOTIENT AND CSF IgM (fig 5 and table I)}

The IgA/albumin quotient was of little value in distinguishing between the various diagnostic groups (fig 5). However, it was interesting to note that two patients with neurosyphilis, one with HSVE and one with a cerebral abscess, had elevated IgA/albumin quotients when compared with the neurological control group. (An upper limit for the IgA/albumin quotient was arbitrarily set at $5 \%$ in order to exclude the upper $2.5 \%$ of specimens of the neurological control group.) It was additionally noted that three other patients with HSVE had high normal values between $4 \%$ and $5 \%$.

CSF IgM was measured in many of the patients, 

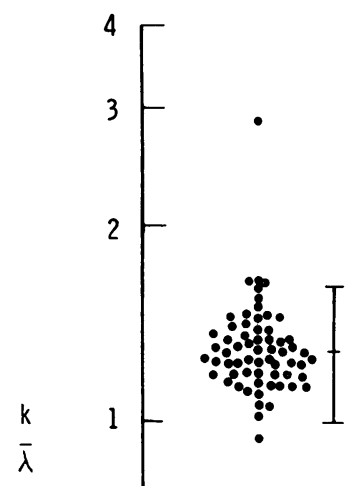

$n=63$
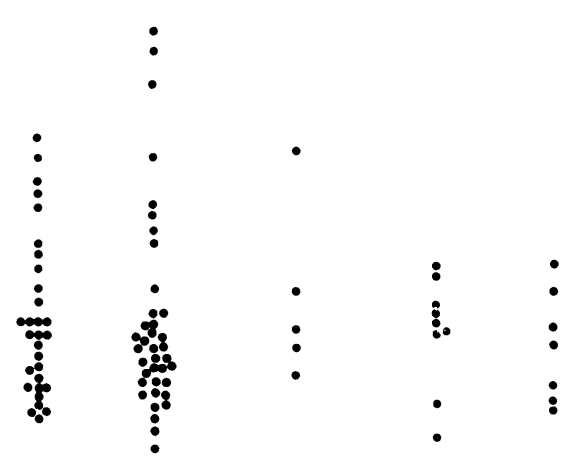

Fig 3 The $\kappa / \lambda$ ratios obtained for the various diagnostic groups.

including all those with MS or neurological infections. IgM was not measurable in the CSF of any patients with MS. Six patients with CNS infection had measurable CSF IgM. These consisted of four patients with HSVE, one with neurosyphilis, and one with tuberculous meningitis. Two patients with polyneuritis also had IgM in the CSF. The relationship between the CSF immunoglobulins and the total proteins for all these patients is shown in table $I$.

\section{Assessment of viral antibodies and a comparison with the CSF immunoglobulin quotients}

A number of CSF and serum specimens were tested for antibodies against three viruses. These results will now be described and a comparison made with the corresponding CSF IgG/albumin quotient and the kappa/lambda ratio.
ANTIBODY AGAINST MEASLES VIRUS

Thirty-two paired serum and CSF specimens from patients with MS or other neurological disorders and an additional 37 CSF specimens from MS patients and 10 from patients with other neurological disorders (all of these 47 latter specimens had elevated IgG/albumin quotients) were examined for measles antibody.

The histogram of the serum measles antibody titre is shown in figure 6. The median for the serum measles titre for the MS group was significantly higher than that for the other neurological disease group; $1: 64$ compared with $1<: 16,0.02<P<$ 0.05 (Wilcoxon sum of ranks statistical test). Seven of these MS CSF specimens had measles antibody with a titre varying from $1: 8$ to $1:<2$ (fig 6). Three specimens in the other neurological disease group also had antibody but with titres less than 1:2. In 


\begin{tabular}{|c|c|c|c|c|c|c|c|c|c|c|c|c|c|}
\hline \multirow[t]{3}{*}{ Patient } & \multirow[t]{3}{*}{ Age } & \multirow[t]{3}{*}{$\operatorname{Sex}$} & \multirow[t]{3}{*}{ Diagnosis } & \multirow{3}{*}{$\begin{array}{l}\text { Cell } \\
\text { count } \\
\left(m m^{-3}\right)\end{array}$} & \multirow{3}{*}{$\begin{array}{l}\text { Total } \\
\text { protein } \\
(m g \%)\end{array}$} & \multirow{3}{*}{$\begin{array}{l}\operatorname{Ig} G / \\
\text { albumin } \%\end{array}$} & \multirow{3}{*}{$\frac{C S F}{\text { serum }} /$} & \multirow{3}{*}{$\frac{\operatorname{Ig} G}{\text { albumin }}$} & \multirow[t]{3}{*}{$\kappa / \lambda$} & \multicolumn{4}{|c|}{ Viral antibody titre } \\
\hline & & & & & & & & & & \multirow{2}{*}{$\begin{array}{l}\text { Serum } \\
\text { measles }\end{array}$} & \multicolumn{3}{|l|}{$C S F$} \\
\hline & & & & & & & & & & & Measles & $H S V$ & Mumps \\
\hline 1 & 31 & $\mathbf{M}$ & Definite & 8 & 104 & 16.4 & 97 & & 2.04 & $1: 128$ & $1: 4$ & - & - \\
\hline 2 & 28 & $\mathbf{M}$ & Definite & 10 & 42 & $18 \cdot 9$ & $74 \cdot 4$ & & 1.5 & $1: 64$ & $1:<2$ & - & - \\
\hline 3 & 21 & $\mathbf{F}$ & Probable & 8 & 58 & $23 \cdot 9$ & 90.6 & & 2.08 & $1: 128$ & $1:<2$ & 一 & - \\
\hline 4 & 23 & $\mathbf{F}$ & Probable & 8 & 51 & $15 \cdot 1$ & $39 \cdot 7$ & & $1 \cdot 12$ & $1: 64$ & $1:<2$ & - & - \\
\hline 5 & 39 & $\mathbf{F}$ & Probable & 5 & 56 & $19 \cdot 4$ & ND & & $2 \cdot 35$ & $1: 64$ & $1:<2$ & - & - \\
\hline 6 & 54 & $\mathbf{F}$ & Probable & 0 & 40 & $20 \cdot 2$ & 106 & & $1 \cdot 77$ & ND & $1: 16$ & - & ND \\
\hline 7 & 63 & $\mathbf{M}$ & Possible & ND & 51 & 30.4 & ND & & $1 \cdot 21$ & ND & $1: 4$ & $1:<2$ & ND \\
\hline 8 & 27 & $\mathbf{F}$ & Definite & 14 & 43 & $31 \cdot 8$ & ND & & $1 \cdot 38$ & ND & $1: 4 / 8$ & $1:<2$ & ND \\
\hline 9 & 42 & $\mathbf{F}$ & Probable & 42 & 158 & $19 \cdot 9$ & ND & & 1.69 & ND & $1: 2$ & - & ND \\
\hline 10 & 55 & $\mathbf{F}$ & Definite & 3 & $57 \cdot 5$ & 35 & 177 & & 1.61 & $1: 128$ & $1: 8$ & - & - \\
\hline 11 & 22 & $\mathbf{M}$ & Definite & 83 & 43 & $42 \cdot 8$ & 189 & & $1 \cdot 5$ & $1: 512$ & $1: 16$ & - & - \\
\hline
\end{tabular}

Table I Patients with MS in which measles antibody was detected in CSF - negative; ND not done.

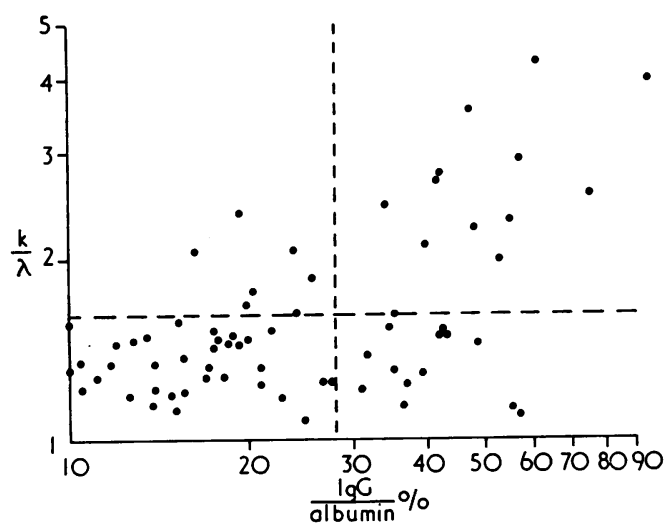

Fig 4 Scattergram of the $C S F \kappa / \lambda$ ratio and the IgG/albumin quotient for 68 patients with MS. The dashed lines indicate the upper limit for the neurological control group. Note the six specimens with normal IgG/albumin quotients but with elevated $\kappa / \lambda$ ratios.

general, patients with detectable CSF measles antibody also had high serum titres; this relationship and the mean age and sex ratio for both groups are shown in figure 6. The CSF total proteins were comparable in both groups.

From the 47 additional CSF specimens screened for measles antibody, four were found (all were from patients with MS) to contain antibody and with a titre varying from $1: 2$ to $1: 16$. Two of these positive specimens also had antibody against HSV (titre 1: $<2$ ), but in neither was the CSF total protein elevated, and both had only slightly elevated IgG/albumin quotients (table I).

The presence of CSF measles antibody in the MS group was variably related to an elevated IgG/ albumin quotient. Seven patients out of the 53 tested had detectable measles antibody associated with normal values for the IgG/albumin whereas only four patients with antibody showed an elevated IgG/albumin quotient (table II). In particular, two patients had high CSF titres of measles antibody $(1: 16,1: 4)$ but normal IgG/albumin quotients. The serum measles titre was $1: 128$ in the second patient (it was not measured in the first) and the CSF total protein was normal in the first patient and elevated to $105 \mathrm{mg} \%$ in the second.

The single patient with SSPE had a grossly abnormal CSF/serum measles titre ratio of $1: 8$ and a CSF total protein of $84 \mathrm{mg} \%$.

\section{ANTIBODY AGAINST HERPES SIMPLEX VIRUS}

The CSF and serum from all nine patients with HSVE and the CSF from 54 patients with MS and from 26 patients with other neurological disorders were tested for antibody against HSV.

Antibody against HSV was detected in the CSF in eight of the nine HSVE patients. In the antibody

\begin{tabular}{|c|c|c|c|c|c|c|}
\hline Patient & Diagnosis & $\begin{array}{l}\text { CSF total protein } \\
(\mathrm{mg} \%)\end{array}$ & IgG/albumin $\%$ & IgA/albumin $\%$ & $\begin{array}{l}\operatorname{Ig} M \\
(m g \%)\end{array}$ & $\kappa / \lambda$ \\
\hline $\begin{array}{l}1 \\
2 \\
3 \\
4 \\
5 \\
6 \\
7 \\
8\end{array}$ & $\begin{array}{l}\text { HSVE } \\
\text { HSVE } \\
\text { HSVE } \\
\text { HSVE } \\
\text { Neurosyphilis } \\
\text { TB meningitis } \\
\text { Polyneuropathy } \\
\text { Polyneuropathy }\end{array}$ & $\begin{array}{r}132 \\
175 \\
224 \\
319 \\
85 \\
367 \\
570 \\
330\end{array}$ & $\begin{array}{r}55 \cdot 0 \\
66 \cdot 1 \\
133 \cdot 0 \\
17 \cdot 3 \\
95 \cdot 6 \\
18 \cdot 6 \\
20 \cdot 3 \\
18 \cdot 4\end{array}$ & $\begin{array}{l}4 \cdot 40 \\
4 \cdot 04 \\
8 \cdot 49 \\
1 \cdot 53 \\
6 \cdot 43 \\
3 \cdot 0 \\
3 \cdot 30 \\
1 \cdot 58\end{array}$ & $\begin{array}{l}0 \cdot 85 \\
0 \cdot 10 \\
1 \cdot 30 \\
0 \cdot 13 \\
0 \cdot 10 \\
3 \cdot 0 \\
0 \cdot 85 \\
0 \cdot 13 \mathrm{~s}\end{array}$ & $\begin{array}{l}1.05 \\
1.51 \\
1.66 \\
1.51 \\
1.81 \\
1.64 \\
1.3\end{array}$ \\
\hline
\end{tabular}

Table II Patients with all three immunoglobulins detected in the CSF 


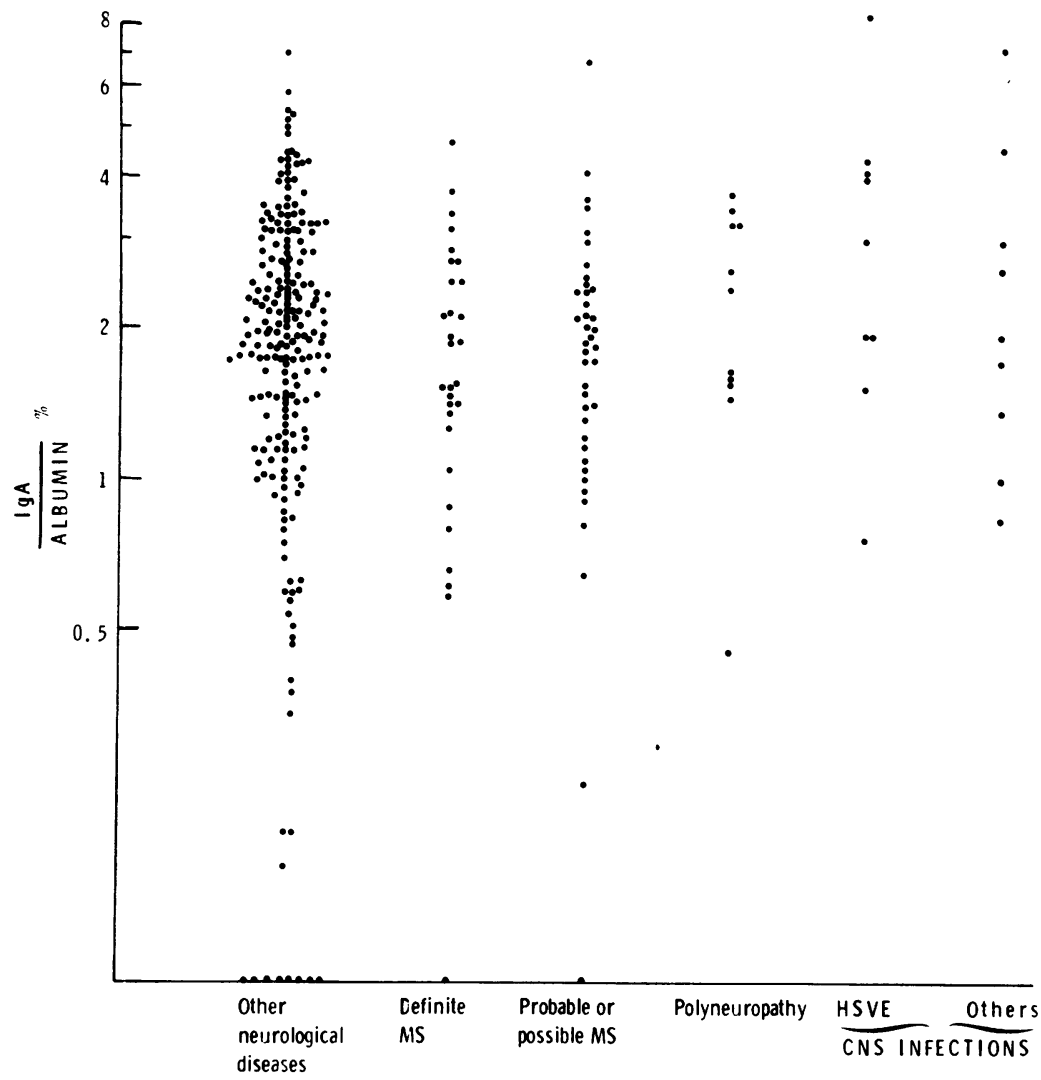

Fig 5 IgA/albumin quotients obtained for the various groups.

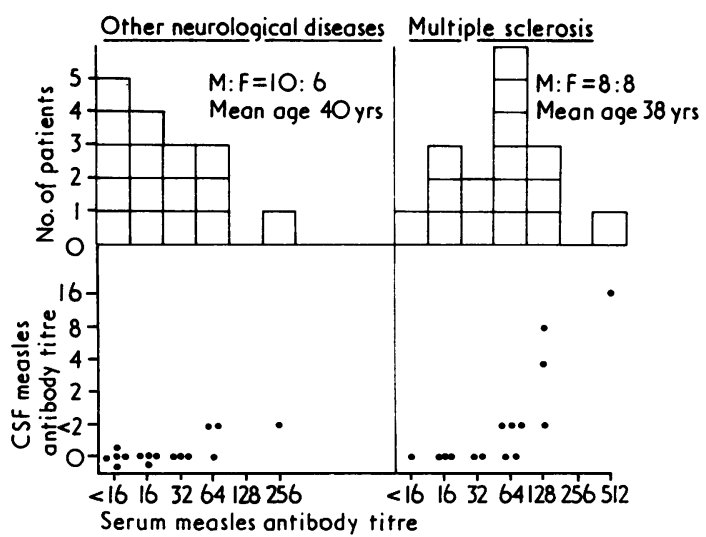

Fig 6 Relationship between serum and CSF titre of measles antibody for a group of patients with definite or suspected MS and for patients with other neurological disorders.

positive patients the ratio of the CSF/serum antibody titres varied between $1: 1$ and 1:16, grossly abnormal values (MacCallum et al, 1974). These viral studies have been reported elsewhere and will not be further discussed (MacCallum et al, 1974).

The IgG/albumin quotient was elevated in six of the antibody positive patients. In addition, two of the remaining three patients had high normal quotients of $27.5 \%$ and $25.6 \%$ (see fig 1). In the former patient an abnormal total protein of 109 $\mathrm{mg} \%$ was recorded while in the other the total protein was normal. The CSF/serum antibody titre was $1: 16$ in the former patient and it was not measured in the latter.

No antibody against HSV was detected in any of the CSF specimens from the neurological control group while two patients with MS had antibody against HSV (vide supra).

One patient with neurosyphilis showed a CSF antibody titre to both HSV $(1: 16)$ and measles virus (1:4). The CSF protein in this patient was $85 \mathrm{mg} \%$ and $25 \%$ of this was IgG.

ANTIBODY AGAINST MUMPS VIRUS

The CSF from 17 patients with MS and from 16 patients with other neurological disorders was examined for mumps antibody. No antibody was detected in any of these specimens. 


\section{Discussion}

The results of this study have confirmed the original observation of Kabat and his colleagues (1948) and subsequent workers that a relative rise in CSF IgG is frequently seen in demyelinating and infecting CNS disorders.

Link and Müller (1971), in a recent comprehensive study, noted a relative increase in CSF IgG in $73 \%$ of 64 patients with definite MS, in $36 \%$ of 39 patients with CNS infections, and in $16 \%$ of 81 patients with other neurological disorders when compared with 30 'healthy' controls. However, in clinical neurological practice it is often not so much a problem of distinguishing normal from abnormal but in distinguishing MS from non-MS or CNS infections from non-infections. We have, therefore, compared a group of patients with CNS infections, MS or polyneuritis with a group comprised of patients with other neurological disorders. The results of this study have shown that the measurement of the relative concentration of IgG in the CSF can be of diagnostic value and help in distinguishing MS and CNS infections from other neurological disorders.

It has also been shown that distribution of the values of the relative CSF IgG, as assessed by the IgG/albumin quotient, obtained for patients with radiologically proven cervical spondylosis or lumbar disc herniation was similar to that obtained for the larger neurological control group. If this smaller group is taken as a 'normal' group, representing the situation seen in healthy individuals, this latter finding suggests that a relative increase in CSF IgG is uncommon in neurological disorders apart from MS and CNS infections. This conclusion is supported by other investigators who have found that between $5.5 \%$ and $22 \%$ of patients with other neurological disorders show elevated values of CSF IgG quotients when compared with essentially healthy subjects (Kabat et al, 1950; Harter et al, 1962; Schneck and Claman, 1969; Riddoch and Thompson, 1970; Link and Müller, 1971). However, it is difficult to compare or contrast individual studies due to the different diagnostic criteria and CSF quotients used in the assessment of the patients.

In many studies a small number of patients with presenile dementia, with or without extrapyramidal features, have been noted to show elevations of the relative CSF IgG concentration (Yahr et al, 1954; Riddoch and Thompson, 1970; Link and Müller, 1971; Skrabanek et al, 1973). In the present study two such patients were observed. The clinical features of one patient were non-specific while the pathological examination of the CNS in the second showed only a cerebral infarct. In neither patient was the serum examined for the presence of a polyclonal or monoclonal increase in IgG which would also increase the CSF IgG/albumin quotient (Riddoch and Thompson, 1970). It would be of interest to know whether such patients have undiagnosed MS, which occasionally gives rise to a presenile dementing process (McAlpine et al, 1972); a CNS infection such as HSV, which has been suggested to be a cause of early dementia (MacCallum, et al, 1974); or whether these patients suffer from another disorder.

The IgG/albumin quotient was elevated in $53 \%$ of the patients with definite MS as compared with $28 \%$ of the patients with suspected MS. The difference between these groups could be due to the inclusion of patients in the latter group who will ultimately turn out to have another disease. But, alternatively, since many of these suspected MS patients were suffering from an initial acute neurological episode, this might suggest that the IgG/ albumin quotient is not elevated in the early stages of MS with the same frequency as is seen for established MS. Follow-up and serial estimations of the IgG/albumin quotient in these suspected MS patients will help to clarify this problem. However, Glasner (1974) has noted a rise in the relative CSF IgG concentration in the first few months after the initial attack in the majority of 96 patients with MS. The relative IgG concentration appeared to stabilize after an interval of one year from the initial episode.

One-third of the definite MS patients in this study had an elevated kappa/lambda ratio when compared with the neurological control group. This figure is a little lower than the $53 \%$ obtained by Link and Müller (1971). These investigators also measured the same ratio in the serum of their patients and found no difference between the groups. They therefore concluded that the increase in the kappa/lambda ratio in the MS CSF was probably due to a disproportionate increase in the quantity of the IgG molecules with light chain of type kappa. Why this should occur is not known.

Viral antibody studies were performed in many of the MS patients. The increased serum title of measles antibody in a group of 16 patients with MS when compared with a group having other neurological disorders, is in accord with previously reported studies (Adams and Imagawa, 1962; Haire et al, 1973).

Norrby et al (1974) have studied measles titres in serum and CSF in 30 patients with definite MS and in 30 patients with other neurological disorders. They found haemagglutination-inhibiting and/or nucleocapsid complement-fixing measles antibody in $13 \mathrm{MS}$ CSF specimens in contrast to their neurological control group in which only two specimens 
were positive. They also found a significant reduction in the serum/CSF ratio of these measles antibodies in 12 of the 30 MS patients but did not see any reduction in their neurological disease group. These investigators conclude that antibody against measles virus is produced in the CNS of some MS patients.

In the present study the examination of $53 \mathrm{CSF}$ specimens from patients with definite or suspected MS revealed antibody in 11 . Seven of these antibody positive patients had a normal IgG/albumin quotient. In the study of Norrby et al (1974) four of their MS patients had a normal IgG/total protein quotient and one of these had a significantly reduced serum/CSF ratio of measles antibody. Both these findings therefore suggest that some patients with MS may have CNS production of measles antibody without any elevation of the relative concentration of CSF IgG. The assessment of CSF measles antibody or, more importantly, the serum/ CSF measles antibody titre may be useful in detecting some patients with MS who have normal IgG/ albumin quotients.

Rosenthal and Soothill (1962) have shown that the concentration of CSF IgG and albumin is dependent on the concentration of these proteins in the plasma. To account for variations in the levels of these plasma proteins the quotient $\mathrm{CSF} /$ serum IgG, expressed as a percentage of the $\mathrm{CSF} /$ serum albumin, was measured in MS and other neurological patients and compared with the IgG/albumin and $\mathrm{IgG} /$ total protein quotient. It was observed that an increased proportion of patients with definite or suspected MS had elevated values of this former quotient when compared with the latter two quotients. Furthermore, in two patients the $\mathrm{IgG} /$ albumin and IgG/total protein quotients were normal but were associated with an elevated $\mathrm{CSF} / \mathrm{serum}$ quotient and with an abnormal $\kappa / \lambda$ ratio and with detectable measles antibody in the CSF. These findings suggested that this latter quotient is more discriminating in detecting patients with MS than the more generally used IgG/albumin or IgG/total protein quotient.

It was of particular interest that 6/9 patients with herpetic encephalitis had marked elevations of the IgG/albumin quotient. These six specimens of CSF were all obtained between the 12th and the 200th day of the illness. Normal quotients were seen in one patient at the second day of the illness and in another who had survived for longer than one year. A rising quotient was also observed in one patient in the early stages of the disease and a falling quotient in another (over a five-month period). These observations are reminiscent of the rise seen in the CSF titre of antibody to HSV in the first two weeks of the disease and the slow decline over the following $\frac{\stackrel{0}{\vec{\sigma}}}{\underline{0}}$ months (MacCallum et al, 1974).

A similar increase in CSF IgG in HSVE has been $\overrightarrow{\vec{F}}$ previously noted by von Welsum and van der Helm $\overrightarrow{0}$ (1970), Rappel et al (1971), and Link and Müller 듬 (1971), but only in the latter study have the CSF $\frac{\bar{\sigma}}{2}$ immunoglobulins been measured quantitatively.

Elevation of the IgG/albumin quotient was also found in the present study in cases of neurosyphilis SSPE, cerebral abscess, and meningitis, as has been $\vec{\circ}$ previously described by others (Yahr et al, 1954; $\overrightarrow{\vec{\omega}}$ Link and Müller, 1971).

Patients with polyneuropathies, including those 응 with the characteristic features of the GuillainBarré syndrome, have been recorded in which a relative increase in the CSF IgG or gamma globulin $\vec{N}$ has occurred (Harter et al, 1962; Laterre et al, 1970; $\vec{\overrightarrow{ }}$ Link, 1973). In general, the frequency of occurrence $O$ is low and compares with the $17 \%$ seen in the pre-을 sent study. The nature or cause of the selective in- crease of CSF IgG is unknown. Link (1973) has also described monoclonal bands occurring in the serum of 8 out of 10 patients with the Guillain-Barré $\frac{\mathbb{S}}{3}$ syndrome similar to that seen for one patient in the present study. The origin and function of this monoclonal immunoglobulin are unknown. $\delta \overrightarrow{0}$

The measurement of CSF IgA and IgM was ngt. of great use in the comparison of the various groups. $\operatorname{IgA}$ and $\operatorname{IgM}$ were relatively increased in some patients with CNS infection but were too few in numbers in these groups for one to decide whether: this was a constant feature. It appeared that $\operatorname{IgA}$ and $\stackrel{\mathcal{Q}}{\mathcal{Q}}$ IgM were not included in the CNS antibody re- $\overrightarrow{\vec{F}}$ sponse seen in MS, and this finding is in keeping 3 with the conclusion of other investigators (Link, 1967; Link and Müller, 1971).

Five patients out of 16 tested with CNS infections showed an elevated CSF $\kappa / \lambda$ ratio while $2 / 5$ patients 0 with polyneuropathy also showed this increase. This is in contrast to the findings of Link and: Müller (1971) where none of the 39 patients witho CNS infections showed any change in this ratio. The present results show that alterations in the $\kappa / \lambda$ ? ratio are not specific for MS.

In conclusion, it is considered that the estimation of the relative concentration of CSF immuno- N globulin, together with other methods of CSF immunoglobulin analysis (including $\kappa / \lambda$ ratio, viral antibody studies, and electrophoresis), will supple- $\omega$ ment each other in the endeavour to detect pathological production of immunoglobulin in the CNSo in certain neurological conditions.

We should like to thank the consultants and staff of the Neurology Department, Churchill Hospital for allowing us to study their patients. PJR-T is a re 
cipient of the Senior Hulme (Overseas) Scholarship, Brasenose College, Oxford. Mrs A. Houghton gave much appreciated secretarial assistance.

We gratefully acknowledge the generosity of $\mathrm{Dr}$ F. O. MacCallum in contributing seven specimens of CSF from patients with HSVE, and his staff for measuring the viral antibody titres.

\section{References}

Adams, J. M. and Imagawa, D. T. (1962). Measles virus antibodies in multiple sclerosis. Proc. Soc. exp. Biol. (N.Y.), 111, 562-566.

Glasner, H. (1974). Gammaglobulins in cerebrospinal fluid during various stages of multiple sclerosis. Excerpta med. (Amst.), Sect. VIII, 32, 657.

Haire, M., Fraser, K. B., and Millar, J. H. D. (1973). Measles and other virus-specific immunoglobulins in multiple sclerosis. Brit. med. J., 2, 612-615.

Harter, D. H., Yahr, M. D., and Kabat, E. A. (1962). Neurological diseases with elevation of cerebrospinal fluid gamma globulin: a critical review. Trans. Amer. neurol. Ass., 87, 210-212.

Humphrey, J. H. and Batty, 1. (1974). International reference preparation for human serum IgG, IgA, IgM. Clin. exp. Immunol., 17, 708.

Kabat, E. A., Freedman, D. A., Murray, J. P., and Knaub, V. (1950). A study of the crystalline albumin, gamma globulin and total protein in the cerebrospinal fluid of 100 cases of multiple sclerosis and in other diseases. Amer. J. med. Sci., 219, 55-64.

Kabat, E. A., Glusman, M., and Knaub, V. (1948). Quantitative estimation of the albumin and gamma globulin in normal and pathologic cerebrospinal fluid by immunochemical methods. Amer. J. Med., 4, 653-662.

Laterre, E. C., Callewaert, A., Heremans, J. F., and Sfaello, Z. (1970). Electrophoretic morphology of gamma globulins in cerebrospinal fluid of multiple sclerosis and other diseases of the nervous system. Neurology, 20, 982-990.

Link, H. (1967). Immunoglobulin $G$ and low molecular weight proteins in human cerebrospinal fluid: chemical and immunological characteristics with special reference to multiple sclerosis. Acta neurol. scand., Suppl, 28, 1-136.

Link, H. (1973). Immunoglobulin abnormalities in the Guillain-Barré syndrome. J. neurol. Sci., 18, 11-23.

Link, H. and Müller, R. (1971). Immunoglobulins in multiple sclerosis and infections of the nervous system. Archives of Neurology, 25, 326-344.

McAlpine, D., Lumsden, C. E., and Acheson, E. D. (1972). Multiple Sclerosis: A Reappraisal, p. 202. Churchill Livingstone, Edinburgh.
MacCallum, F. O., Chinn, I. J., and Gostling, J. V. T. (1974), Antibodies to herpes-simplex virus in the cerebrospinal fluid of patients with herpetic encephalitis. J. med. Microbiol., 7, 325-331.

Mancini, G., Carbonara, A. O., and Heremans, J. F. (1965). Immunochemical quantitation of antigens by single radial immunodiffusion. Immunochemistry, 2, 235-254.

Norrby, E., Link, H., and Olsson, J. (1974). Measles virus antibodies in multiple sclerosis. Arch. Neurol., 30, 285-292.

Rappel, M., Dubois-Dalcq, M., Sprecher, S., Thiry, L., Lowenthal, A., Pelc, S., and Thys, J. P. (1971). Diagnosis and treatment of herpes encephalitis. J. Neurol. Sci., 12 , 443-458.

Riddoch, D. and Thompson, R. A. (1970). Immunoglobulin levels in the cerebrospinal fluid. Brit. med. J., 1, 396-399.

Rosenthal, F. D. and Soothill, J. F. (1962). An immunochemical study of the proteins in cerebrospinal fluid. J. Neurol. Neurosurg. Psychiat., 25, 177-181.

Schneck, S. A. and Claman, H. N. (1969). CSF immunoglobulins in multiple sclerosis and other neurologic diseases. Measurement by immunodiffusion. Arch. Neurol., 20, 132-139.

Schultze, H. E. and Heremans, J. F. (1966). Molecular Biology of Human Proteins, Vol. I, pp. 732-761. Elsevier, Amsterdam.

Skrabanek, P., Holland, P. D. J., Staunton, H., and Lawlor, L. (1973). Immunoglobulins in CSF in multiple sclerosis and other neurological disorders. J. Irish med. Ass., 66, 692-696.

Smith, I. W., Peutherer, J. F., and MacCallum, F. O. (1967). The incidence of Herpes virus hominis antibody in the population. J. Hyg. (Camb.), 65, 395-408.

Tourtellotte, W. (1970). On cerebrospinal fluid immunoglobulin-G (IgG) quotients in multiple sclerosis and other diseases. J. Neurol. Sci., 10, 279-304.

Waddell, W. J. (1956). A simple ultraviolet spectrophotometric method for the determination of protein. J. Lab. clin. Med., 48, 311-314.

Weeke, B. and Krasilnikoff, P. A. (1970). A polynomial expression for the serum concentrations of 21 serum proteins from 1-93 years of age in normal females and males. Protides biol. Fluids, 18, 173-179.

van Welsum, R. A. and van der Helm, H. J. (1970). The protein composition of the cerebrospinal fluid in acute necrotizing encephalitis. Neurology, 20, 996-1001.

Yahr, M. D., Goldensohn, S. S., and Kabat, E. A. (1954). Further studies on the gamma globulin content of cerebrospinal fluid in multiple sclerosis and other neurological diseases. Ann. N. Y. Acad. Sci., 58, 613-624.

Zettervall, O. and Link, H. (1970). Electrophoretic distribution of kappa and lambda immunoglobulin light chain determinants in serum and cerebrospinal fluid in multiple sclerosis. Clin. exp. Immunol., 7, 365-372. 